\section{Les Conflits Interethniques en République Démocratique du Congo et Dans la Région des Grands Lacs Africains : Le Cas de L'ituri}

\section{Jok Oga Ukelo*}

*Jok Oga Ukelo est originaire la ville de Kinshasa en République démocratique du Congo. Il est diplômé d'une licence en science politique et administration de l'Université de Kinshasa. M. Ukelo a participé au colloque Bonne gouvernance et participation populaire, organisé par l'organisme Médias pour la paix en République démocratique du Congo en 2001. Il s'intéresse particulièrement à la question du développement humanitaire en République démocratique du Congo.

\section{Abstract}

Black Africa has become the theatre of everlasting war. These are wars in which Africans massacre their brothers and plunder the natural resources of their continent. We can cite the cases of the Bakongo and the Bangala in the Congo Brazzaville or the Tutsis and the Hutu in Rwanda. In this article the questions surrounding the interethnic conflicts in the Democratic Republic of Congo, via a case study of the conflict between the Hema and Lendu in the Ituri region, are explored. The main thrust of the article is that the concept of good governance is linked to a sustainable developmental strategy and that this is the only way to reach peace in this geographical area.

L'Afrique noire est devenue le théâtre de guerres interminables. Des guerres où les Africains massacrent leurs frères. Des guerres consécutives aux conflits armés et interethniques où les fils de ce continent se livrent à un pillage éhonté des richesses naturelles et minières. Nous pouvons citer les cas des conflits armés et interethniques opposant les Bakongo et les Bangala au Congo Brazzaville, les Tutsis et les Hutus au Rwanda. En fait, dans cet essai, il sera question des conflits interethniques en République démocratique du Congo et dans la région des Grands Lacs par l'entremise d'une étude de cas : les conflits entre Hema et Lendu en
Ituri. L'argumentation principale se fera en lien avec le concept de bonne gouvernance politique et de développement humain durable qui sont pour l'auteur les deux seules avenues possibles de pacification

Dans un premier temps, les conflits interethniques découlent d'une opposition d'intérêts entre deux ou plusieurs ethnies, née du fait de leur appartenance à des origines, à des langues, à des coutumes et à des vocations différentes, réprimés et déformés par des modèles préconçus et des théories bâties de toutes pièces par des systèmes socio-politiques biaisés. Cet antagonisme est caractérisé par des affrontements, des violences, des guerres et des tueries entre des ethnies différentes. Cela est justifié par des revendications de territoires, de reconnaissance identitaire, de reconnaissance de droits, de dignités et de participation à la vie politique de la part d'ethnies qui se croit soit dominées, soient dominantes ou croyant avoir plus de droits, de privilèges et d'obligations comparativement à d'autres.

En Afrique, l'ethnicité joue un rôle majeur dans la dialectique entre l'homme et la société. On ne peut donc pas expliquer le phénomène politique sans se référer d'une manière ou d'une autre au phénomène ethnique. L'ethnicité est une identité, un sentiment qui joue sur l'affectivité de l'individu à autrui. L'ethnicité est aussi une idéologie, c'est-à-dire : " un ensemble de croyances, d'idées et de valeurs fondé sur le sens actif de l'appartenance à une unité ethnique que l'on trouve chez tous les Africains ; que ce groupe soit structuré ou non. ${ }^{1}$ L'ethnicité est une arme et une stratégie politique. A titre d'exemple, lors des élections ou des prises de position, on mobilise les frères de tribu avec une argumentation simple telle votez-moi ou soutenez-moi parce que je suis votre frère de tribu. Ce genre de parole affective frappe directement le cœur. L'ethnicité est aussi le fruit d'un mensonge idéologique et d'une construction identitaire de la part des colonisateurs ayant comme objectif de mener à bien leur politique « diviser pour mieux régner ». Relevons aussi que certaines ethnies, en dépit des mutations socio-politiques causées par des évènements traumatiques tel que la conquête, l'esclavage, la dispersion et la conversion religieuse, maintiennent toujours un sens propre à leur continuité identitaire. De ce qui précède, nous pouvons observer que l'ethnicité a plus une dimension subjective qu'objective, car elle fait appel au sentiment d'appartenance et non à la raison universelle.

${ }^{1}$ Lumuna KABUYA, Cours de Sociologie Politique, Première Licence Sciences Politiques et Administratives, Université de Kinshasa, 2003. 
Par ailleurs, les conflits armés et interethniques entravent les relations politique et économique entre les acteurs africains à la suite de la haine, de la déstabilisation, des frustrations et des destructions qu'ils provoquent. Cette situation a plongé plusieurs ethnies, surtout en Afrique, dans une phobie Kafkaïenne où les massacres, les tueries et les guerres deviennent une fatalité et une tragédie caractérisées par une terreur que les chercheurs et les hommes de science, la communauté tant nationale qu'internationale observent sans un réel empressement d'intervention afin d'y mettre un terme. Ces conflits et leurs conséquences constituent donc un défi fondamental pour l'Afrique. En vérité, négliger de rechercher de nouvelles réponses aux problèmes omniprésents découlant des conflits armés revient à condamner les peuples d'Afrique à vivre dans des conditions où la guerre devient une malédiction qu'il faut endurer dans des conditions de vie exécrables et non un problème qu'il faut résoudre définitivement. $^{2}$

Dans un deuxième temps, les conflits armés et interethniques n'ont pas épargné la République Démocratique du Congo. L'Ituri, l'un de quatre districts qui constituent la Province Orientale compte environ 2570000 habitants parmi lesquels on trouve les principaux groupes ethniques d'Afrique noire (Pygmées, Bantous, Soudanais, Amites et Nilotiques). Cette zone géographique couve depuis très longtemps un conflit interethnique opposant l'ethnie Hema (éleveur) et l'ethnie Lendu (agriculteur). Ce district, situé au Nord-Est de la République Démocratique du Congo, fait frontière avec le Soudan au Nord, l'Ouganda à l'Est, la Province du Nord Kivu au Sud et les districts de la Tshopo et du Haut Uélé à l'Ouest.

D'une part, les Hema sont éleveurs et commerçants. Le bétail joue un grand rôle dans la société Hema. Le prestige et le rang social sont déterminés par le nombre de têtes de bétail que possède un individu. $\mathrm{Ce}$ bétail constitue un élément de thésaurisation, car il sert de valeur dotale et d'échange. Il acquiert petit à petit, au fur et à mesure de l'évolution de la société, une valeur marchande. L'une des raisons de la migration Hema fut la recherche de vastes pâturages pour le bétail.

D'autre part, les Lendu sont sédentaires, ce qui est l'apanage des agriculteurs. Ils aiment cultiver la terre et s'enorgueillissent de posséder de grandes cultures et de récolter en grandes quantités. Ils se servent de la houe pour cultiver leur champ. Dans chacune de ces opérations, la houe a une valeur instrumentale et une valeur symbolique. Dès lors, l'introduction d'un

\footnotetext{
2 Amoo G. SAM, Le défi de l'ethnicité et des conflits en Afrique : Nécessité d'un
} nouveau modèle, PNUD, New York, 1997, p. 2 et 3 tout autre instrument de labourage (pioche, pelle,...) était mal accueillie par la population convaincue que le changement ne pouvait que nuire à la fécondité des champs et aussi à la qualité et à la quantité des récoltes.

Depuis 1999, ce conflit a pris une autre dimension avec l'occupation de la République démocratique du Congo par les forces armées étrangères (du Burundi, de l'Ouganda et du Rwanda). D'aucuns n'ignorent combien des vies humaines ont été sacrifiées au cours des tueries entre ces deux ethnies. Mais il sied de souligner que cette fois-ci l'ampleur des massacres qui y ont été perpétrés battent tous les records connus dans l'histoire des relations entre les deux ethnies, avec pour conséquences ; des milliers de blessés et de personnes déplacées, plusieurs concessions endommagées et d'importantes pertes matérielles.

Dans un autre temps, en nous livrant à l'analyse du conflit interethnique entre Hema et Lendu, nous nous sommes proposés d'appréhender d'abord le contexte historique du dit conflit pour rechercher les causes lointaines et immédiates qui le font resurgir périodiquement. Cela va nous permettre de bien saisir le rapport des événements qui se sont succédés et de proposer les recommandations pouvant conduire à la recherche d'une solution pacifique et durable à ce conflit.

Compte tenu de notre problématique, plusieurs éléments de causalités peuvent être identifiés. Premièrement, nous croyons qu'il existe un antagonisme né des vocations opposées entre les Hema et les Lendu. Tous se situant dans le district de l'Ituri, les uns sont éleveurs et les autres agriculteurs. Les Hema, éleveurs, sont toujours en quête d'espace supplémentaire pour l'élevage de leurs bétails. La propension à acquérir davantage de territoire rencontre de la résistance de la part des Lendu, agriculteurs tournés vers la protection des terres que tentent d'envahir les éleveurs. Cette rivalité serait à la base de la résurgence des conflits.

Deuxièmement, la colonisation belge a laissé des traces négatives de domination dans les structures administratives. Ainsi, la discrimination initiée par la politique coloniale belge et entretenue par les églises et les missionnaires en Ituri, n'a poussé qu'à la négation de l'autre par la création d'un esprit d'intolérance entre les différentes communautés ethniques. La politique coloniale belge a instauré l'absence de la liberté d'opinion et de la participation de certaines communautés à la gestion de l'espace publique. Ceci a eut pour conséquence d'entretenir des tensions entre les ethnies qui sont favorisées et donc dominantes et celle dominés. Cette situation empêche les différentes communautés ethniques de la région d'effectuer les compromis nécessaires afin de stabiliser leurs relations politiques et 
économiques, les uns cherchant l'intégration, les autres voulant conserver leur pouvoir de domination.

De plus, le mensonge idéologique que subissent les communautés Hema-Lendu de la part de leurs élites personnalités, en quête de leadership et de repositionnement sur la scène politique est un autre problème qui n'a fait que créer un déséquilibre entre l'État et la société civile dans la mesure que ces personnalités ont bloqué différentes initiatives de réconciliation entreprises. En effet, il a été observé que les églises, les élites, personnalités politiques et hommes de science, à qui l'on reconnaît les compétences de délibération, de l'écriture et de la décision, n'ont pas toujours eu un discours conciliateur. C'est au travers de la manipulation de l'ethnicité qu'ils ont essayé de couvrir leurs vrais agendas politiques et économiques. Le fondement de leurs démarches était souvent bâti sur des sentiments et des considérations subjectifs qui ont tous conduit à la xénophobie et à la haine ethnique.

De surcroît, la politique de «diviser pour régner» organisée, entretenue et matérialisée par les troupes étrangères présentes en Ituri du fait de la guerre, a permis d'envenimer les relations entre Hema et Lendu en vue de l'exploitation et du pillage des ressources minières de l'Ituri. Du reste, quant aux troupes étrangères présentes dans ce conflit, soit des groupes armés ou des mouvements de libération de pays limitrophes, le Panel des Experts de l'ONU qui a enquêté sur l'exploitation illégale des ressources naturelles de la RDC a réduit le conflit en Ituri à la lutte pour l'accès au contrôle des réserves naturelles. Selon ce Panel, les Présidents du Rwanda et de l'Ouganda sont sur le point de devenir les parrains de l'exploitation illégale des ressources naturelles et de la continuation du conflit en République Démocratique du Congo, particulièrement en Ituri.

Certes, les constatations effectuées ci-dessus démontrent l'interaction de plusieurs dynamiques entretenant la perpétuation du conflit en Ituri. Cependant, il se dégage le constat selon lequel le conflit interethnique entre Hema et Lendu porte principalement sur la gestion des terres. En d'autres termes, tous les événements de ce conflit ont trait à l'occupation légale ou anarchique des terres ou des concessions foncières par les Hema et les Lendu.

En ce sens, la perception du conflit se pose différemment et selon divers plan selon que les membres de la communauté soient Hema ou Lendu. Effectivement, selon la communauté Hema, les concessions acquises par les Hema ont toutes fait l'objet d'un dossier régulier. Par conséquent, il est inadmissible que le motif lié aux concessions réclamées de certains Hema situés dans la collectivité des Lendu soit la cause immédiate d'agression de tous les Hema et ce de façon généralisée et simultanée.

A l'opposé, selon la communauté Lendu, le conflit foncier concerne l'agrandissement des concessions des Hema à travers l'espace qu'occupe les Lendu sans les consulter au préalable et concerne également la rétrocession sans condition de leurs villages incorporés arbitrairement et brutalement dans la collectivité secteur des Bahemea-sud, secteur Hema.

\section{Bonne gouvernance}

Face à cette situation, nous osons croire qu'une gestion publique qui tient compte de paramètres d'une bonne gouvernance tels que la participation et l'équité, qui ne tente pas de déformer les réalités africaines, pourra réellement et objectivement se pencher sur la satisfaction des besoins ontologiques d'un groupe ${ }^{3}$. Nous citons les besoins universels d'identité, de reconnaissance, de sécurité, de dignité et de participation à la gestion de l'administration publique. Faute de quoi, cette gestion publique ne saurait ni prévenir, ni éviter et ni résoudre les conflits armés et interethniques.

En effet, la bonne gouvernance implique dans la société la réhabilitation de certaines valeurs. Nous citons la liberté d'opinion et la participation du peuple dans la gestion de l'administration publique. La bonne gouvernance implique aussi l'instauration de certains principes tels que l'équilibre entre gouvernants et gouvernés, dans son sens large, et l'équilibre entre le pouvoir et le contre pouvoir, c'est-à-dire entre l'État et la société civile.

En d'autres termes, qui dit bonne gouvernance dit politique publique dans la mesure où elle fait appel à un processus d'élaboration et d'évaluation des structures conçues et adaptées en fonction d'une administration de service, sous réserve des aspirations des différentes communautés. Nulle n'est besoin de démontrer à quel niveau les administrations d'Afrique centrale ont perdu le contrôle face aux problèmes qui leurs sont posés. La longue liste des problèmes de développement des pays de la région des Grands Lacs dénote une crise et une incapacité du pouvoir public. Le pouvoir ici entendu comme l'art et la capacité de diriger et de réguler à tous les niveaux les affaires d'une nation. On assiste plutôt à une personnalisation de la vie politique qui contraint les acteurs au clientélisme, s'ils veulent rester au pouvoir. Les hommes d'État s'arrogent

\footnotetext{
${ }^{3}$ Jacques EPEE GAMBWA, Cours d'analyse des systèmes politiques et sociales, Deuxième Licence Sciences Politiques et Administratives, Université de Kinshasa,
} $2 \mathrm{OO} 3$. 
de vastes pouvoirs discrétionnaires tout en excellent dans la tyrannie et l'arbitraire.

\section{Développement durable}

Au-delà de ce qui précède, il y a lieu de se poser la question de savoir par quel mécanisme les États de la région des Grands Lacs pourront sortir de manière générale ou particulière de cette situation d'impasse ? Afin d'éviter l'aggravation des inégalités entre les communautés et entre les États de la région des Grands Lacs, les administrations de cette région doivent cesser d'être des appareils de répression pour devenir des administrations de service dotées des moyens financées et matériels suffisant, d'un personnel motivé, compétent, efficace et honnête. En ce sens, une carence éthique est observée et démontrée à tous les niveaux par les chefs africains de la région des Grands Lacs. Cette situation interpelle plus d'un, et oblige à repenser une nouvelle Afrique et à répartir sur des nouvelles bases éthiques sans lesquelles l'Afrique continuera à patauger dans le cycle infernal des guerres et des violences ${ }^{4}$.

D'ailleurs, ce cycle infernal de guerres et de conflits armés ne peut en aucun cas favoriser le développement de la région des Grands Lacs et de ses populations. Le développement se comprend ici comme un effort multidimensionnel et constant ${ }^{5}$. Il est la clé de tout épanouissement et de progrès. Bien entendu, il existe des paramètres élémentaires qu'il faut absolument réunir tels que la paix et la sécurité des personnes ainsi que de leurs biens. La revue Congo Afrique, parlant du Nouveau Partenariat pour le Développement de l'Afrique (NEPAD) dans son numéro 378 d'octobre 2003, dit ce qui suit: «À la différence des initiatives en matière de développement qui l'ont précédé (Plan d'action de Lagos, Initiative des Nations Unies pour l'Afrique...), le NEPAD intègre, au-delà des aspects économiques, des éléments propres à conférer une durabilité au processus du développement. Ces éléments sont: la gestion et le règlement des conflits ; les droits de l'homme, la démocratie et la bonne gouvernance. » ${ }^{6}$

Au-delà du caractère multidimensionnel et constant, le développement doit s'inscrire dans la durée c'est-à-dire qu'il doit être durable. En d'autres termes, le développement durable consiste à satisfaire

\footnotetext{
${ }^{4}$ Sylvain TSHIKOJI MBUMBA, De la bonne gouvernance: Appel à un nouvel ordre éthique du pouvoir en Afrique noire, Editions du Cerdaf, 2002, p.21

${ }^{5}$ Olofio BEN OLOMI, Cours d'Economie de Développement, Troisième Graduat Sciences Politiques et Administratives, Université de Kinshasa, 2003

6 «Mythe ou réalité du Développement durable», revue Congo Afrique, numéro 381, Janvier 2004.
}

les besoins des générations précédentes sans compromettre la capacité des générations futures à satisfaire leurs propres besoins. Le développement durable exige donc des sentiments et des comportements de solidarités et de responsabilités. C'est ici qu'intervient le sens du bien commun et le recours au droit afin de rétablir la justice et la paix partout où elles se trouvent menacées.

Enfin, cette démarche devra s'inscrire dans un processus qui exige, à tous les niveaux, des comportements cohésifs et adhésifs des communautés concernées. Ces dernières seront soumises à un même cadre politico-juridique dans lequel elles développeront les mécanismes de participation à la réalisation de l'intérêt tant du district de l'Ituri que de toute la nation congolaise et aussi de toute la région des Grands Lacs. D

\section{Bibliographie}

BEN Olomi, Olofio. 2003. Cours d'Économie de Développement, Troisième Graduat Sciences Politiques et Administratives, Université de Kinshasa,

EPEE GAMBWA, Jacques. 2003. Cours d'Analyse des Systèmes Politiques et Sociales, Deuxième Licence Sciences Politiques et Administratives, Université de Kinshasa, 2003.

TSHIKOJI, Mbumba, Sylvain. 2002 De la bonne gouvernance: Appel à un nouvel ordre éthique du pouvoir en Afrique noire, Kinshasa, Editions du Cerdaf, $160 \mathrm{p}$

«Mythe ou réalité du Développement durable », revue Congo Afrique, numéro 381, Janvier 2004.

« Nouveau Partenariat pour le Développement de l'Afrique (NEPAD)» revue Congo Afrique, numéro 378, Octobre 2003 